\title{
MOTIVACIÓN Y SEGMENTACIÓN DEL TURISTA EN FESTIVALES CULINARIOS. EL CASO DE LA FERIA GASTRONÓMICA EN EL PARQUE NATURAL SIERRAS DE CAZORLA, SEGURA Y LAS VILLAS (ESPAÑA)
}

\author{
Guzmán Antonio Muñoz Fernández ${ }^{1}$ \\ Área de Organización de Empresas \\ Facultad de Derecho y Ciencias Económicas y Empresariales \\ Campus de Excelencia Internacional en Agroalimentación, ceiA3 (Universidad de Córdoba) \\ guzman.munoz@uco.es \\ Jesús Claudio Pérez Gálvez² \\ Área de Economía Aplicada \\ Facultad de Derecho y Ciencias Económicas y Empresariales \\ Campus de Excelencia Internacional en Agroalimentación, ceiA3 (Universidad de Córdoba) \\ dt1pegaj@uco.es \\ Tomás López-Guzmán ${ }^{3}$ \\ Área de Economía Aplicada \\ Facultad de Ciencias del Trabajo \\ Campus de Excelencia Internacional en Agroalimentación, ceiA3 (Universidad de Córdoba)
} tomas.lopez@uco.es

(Fecha envío: 08/11/14 - Fecha aceptación: 28/11/14)

\section{Resumen}

Asociado al espacio natural y al disfrute gastronómico, la Feria del Parque Natural Sierras de Cazorla, Segura y Las Villas (España) expone y comercializa productos agroalimentarios elaborados artesanalmente en este medio natural. Este artículo presenta una investigación que segmenta los turistas a partir de tres dimensiones motivacionales. Los resultados del estudio evidencian que la gastronomía es la principal dimensión motivacional. En base a las dimensiones detectadas se establecen cuatro tipologías de visitantes (turista multimotivado, turista hedónico-gastronómico, turista gastronómico y turista con otras motivaciones) que se consideran como válidas y de utilidad para segmentar el mercado. Las conclusiones muestran que junto a la gastronomía coexisten otros factores motivacionales relevantes que deben ser tenidos en consideración por los organizadores de las ferias gastronómicas.

\section{Palabras clave}

festivales gastronómicos, motivación, segmentación, Parque Natural Sierras de Cazorla, Seguras y la Villas, España

\begin{abstract}
Associate countryside and gastronomic enjoyment, Fair Natural Park Sierras de Cazorla, Segura and the Villas (Spain) states and markets food products handmade in the wild. This paper presents research that targets tourists from three motivational dimensions. The study results show that the food is the main motivational dimension. Based on the dimensions identified four types of visitors (multimotivado tourist, tourist hedonic-gastronomic, tourist and gastronomic tourism with other motivations) that are considered as valid and useful to segment the market provide. The findings show that the cuisine coexist with other relevant motivational factors that must be taken into consideration by the organizers of the food fairs.
\end{abstract}

\section{Key words}

gastronomy festivals, motivation, segmentation, Natural Parks of Cazorla, Segura and Las Villas, Spain

\section{INTRODUCCIÓN}

Las zonas rurales españolas se enfrentan a un lento declive debido a la falta de competitividad de sus productos en unos tiempos donde la estandarización y la globalización se imponen, y solo la cada vez más entredicha atención de las administraciones públicas hace que esta situación no se agudice. Por tanto, es necesario revertir esta situación movilizando los recursos locales e impulsando instrumentos que fomenten el desarrollo social y

\footnotetext{
${ }^{1}$ Doctor en Ciencias Económicas y Empresariales

2 Doctor en Ciencias Económicas y Empresariales

${ }^{3}$ Doctor en Ciencias Económicas y Empresariales
} 
económico local (Stilling y Halkier, 2014). Entre esos instrumentos se encuentra la potenciación de su diferenciación mediante la creación de una "marca local" de sus productos gastronómicos y artesanos. Esta tendencia a la diferenciación a través de la marca se está generalizando, y una de sus mejores acciones de comercialización gira alrededor de los festivales y ferias de productos gastronómicos y de artesanía, donde tanto se venden como se dan a conocer los recursos naturales y culturales locales. Por otro lado, el consumo de productos gastronómicos no es sólo un medio para generar ingresos, sino también es una parte imprescindible de una experiencia turística (Hjalager y Richards, 2002) y un instrumento de diferenciación de las localidades (Sims, 2009), que influye en la intención de visita a un destino (Sedmak y Mihalic, 2008), al esperar el visitante esa comida y ese servicio "típico".

También el desarrollo social, los nuevos hábitos de vida y de trabajo hacen que se busquen nuevas formas de diversión y de expansión que influyen en la necesidad de organizar eventos atractivos como parte de la oferta turística de un lugar o comarca (Marko et al., 2013). Por tanto, los festivales sobre la gastronomía están desempeñando un papel importante en las estrategias de desarrollo rural y de marca que giran en torno a la mercantilización de sus productos locales (Kneafsey, 2007), atrayendo a un importante número de turistas en relación con el tamaño de la localidad, y dando una razón para visitar esa zona. Además, estos festivales sirven para proporcionar trabajo o negocio a los habitantes de estas localidades, dar a conocer la imagen y la marca de sus productos y como elemento identitario para sus habitantes. El identificar las motivaciones turísticas para asistir a este tipo de eventos, así como el grado de satisfacción, es determinante para que las diferentes administraciones públicas puedan desarrollar una estrategia de marketing eficaz y centrar sus esfuerzos en el turista potencial.

El objetivo de este artículo es presentar los resultados de una investigación empírica realizada en la Feria Gastronómica y Artesanal del Parque Natural Sierras de Cazorla, Segura y Las Villas celebrado en Pozo Alcón (Jaén) en octubre de 2014, festival que aspira a convertirse en un referente en gastronomía tradicional en España y que cada año atrae a turistas con la finalidad de degustar, en un entorno natural, los productos típicos de esta zona geográfica, caracterizados por una importante apuesta por las tradiciones culinarias. Así, y en el apartado de los resultados de la investigación de este artículo, se presenta el perfil sociodemográfico, los motivos de la visita y una segmentación de los turistas en función de diversas dimensiones motivacionales detectadas. Para conseguir estos objetivos el artículo se estructura, tras esta introducción, en un segundo apartado donde se realiza una revisión de la literatura en el campo del gastroturismo; en el tercer apartado se muestra la metodología utilizada en la investigación; en el cuarto se presenta los principales resultados de la investigación. El artículo finaliza con las conclusiones y las referencias bibliográficas utilizadas.

\section{REVISIÓN DE LA LITERATURA}

Se puede definir el turismo gastronómico, siguiendo a Ignatov y Smith (2006), como una tipología de turismo en el cual se compra o consume productos regionales (incluyendo bebidas) y/o se observa y se participa en la producción de alimentos (abarcando desde la agricultura hasta las escuelas de cocina). Existe una amplia variedad de términos, sobre todo en inglés, que tratan de vertebrar la relación que existe entre la cocina y el turismo. Entre otros destacamos los siguientes: cuisine tourism, food tourism, gastronomy tourism, gourmet tourism, gastronomic tourism o culinary tourism. Asimismo, en español también existen diferentes conceptos que abordan esta temática tales como gastroturismo, turismo gastronómico o turismo culinario. La literatura académica en el campo del gastroturismo es amplia y extensa, habiendo tenido un incremento significativo en los últimos años. Como publicaciones de referencia en el campo del gastroturismo encontramos las siguientes: la obra de Hjalager y Richards (2002), la aportación de Boniface (2003), el trabajo de Long (2004), las investigaciones de Cohen y Avieli (2004) y las dos obras coordinadas por el profesor Hall y centradas en gastronomía (Hall et al., 2003) como en el vino (Hall et al., 2000).

La literatura científica (Schülter, 2006; Okumus et al., 2007; Tikkanem, 2007; Oliveira, 2007; McKercher et al., 2008; López-Guzmán y Sánchez Cañizares, 2012) documenta y refuerza la idea de la estrecha relación que existe entre la gastronomía y el turismo. Así, y siguiendo a Tikkanem (2007), la relación que existe entre la gastronomía y el turismo se puede vertebrar en cuatro variables diferentes: primera, como atracción, lo cual significa que el destino puede utilizar la gastronomía como un recurso para promocionar y publicitar dicha área geográfica; segunda, como un componente más del producto, donde se ahonda en el diseño de rutas gastronómicas; tercera, como experiencia, abordando la existencia de uno o varios lugares donde la gastronomía adquiere un nivel de excelencia y se convierte en un reclamo en sí mismo; cuarta, como fenómeno cultural, sustentándose en la existencia de diferentes festivales culinarios. Por tanto, el turismo gastronómico tiene un mayor o menor grado de desarrollo de acuerdo con el nivel de conexión que la cocina tenga con la cultura y con los recursos naturales del lugar donde se halle radicado (Riley, 2005). Ello refuerza la necesidad que tiene esta tipología de turismo de reforzar la apuesta por los ingredientes locales, por el continuo aprendizaje de los actores locales, por la apreciación por su propio consumo y por la importancia de los recursos culinarios autóctonos. En este sentido, en los últimos años han aparecido diferentes festivales gastronómicos ubicados en lugares alejados de los tradicionales flujos de turista y que, gracias a la autenticidad de su cocina, a su calidad y a la diferenciación de su elaboración, está consiguiendo posicionarse como lugares como un cada mayor flujo 
turístico y con un incipiente desarrollo turístico que, como es el caso del festival analizado, complete y refuerce la oferta turística de esta zona geográfica.

La creación de los festivales gastronómicos tiene su fundamentación en que la gastronomía puede ser la motivación principal para visitar un determinado lugar geográfico o una motivación secundaria (pero importante) para viajar a ese determinado lugar. En este sentido, y siguiendo a Quan y Wang (2004), la experiencia que el turista obtiene en estos festivales gastronómicos se podría clasificar en tres grupos diferentes: por contraste (u oposición), por intensificación y por extensión. Así, en el primer caso, por contraste se produce cuando el turista durante su viaje disfruta de una gastronomía completamente diferente a la del lugar de su origen; sería de intensificación cuando refuerza los alimentos que generalmente ingiere en su lugar de residencia; y sería de extensión cuando se alimenta de lo que habitualmente come en su lugar de origen. En este sentido, consideramos que la asistencia a la Feria Gastronómica y Artesanal del Parque Natural Sierras de Cazorla, Segura y Las Villas refuerza la experiencia del turista por intensificación debido a que la mayoría de los productos que se ofertan en dicho festival son habitualmente consumidos por los turistas, pero obviamente, reforzándose, y mucho, la calidad del producto degustado en dicho festival.

La cocina se está configurando como un elemento clave en la promoción y en la consolidación de los destinos turísticos. Así, los destinos turísticos deben de considerar la importancia de la comida como contribución a la experiencia única que el viajero quiere encontrar como parte de la expresión cultural del área geográfica que visita. Y así la cocina puede utilizarse para satisfacer al viajero, para contribuir a la autenticidad del destino y para incrementar el impacto socioeconómico del turismo en la comida local (Du Rand et al., 2003). Recordemos que un destino turístico es algo más que un conglomerado de recursos naturales, culturales o artísticos, ya que tiene también que sorprender en cuanto a la experiencia que busca el turista (Cracolici y Nijkamp, 2008). Y todo ello teniendo en cuenta que los viajeros buscan un equilibrio entre lo predecible y lo nuevo (y diferente), es decir, entre lo seguro y la novedad (Tse y Crotts, 2005). Sin embargo, sí es necesario articular una serie de recursos turísticos en el destino para conseguir desarrollar el turismo culinario. En este sentido, los festivales gastronómicos refuerzan todas estas características, para lo cual deben de presentar una diferenciación clara de sus recursos gastronómicos, que debe de tener una cocina que sea reconocible por parte de los viajeros y que el festival tenga un número variado e importante de establecimientos donde los viajeros puedan disfrutar de esa gastronomía.

\section{METODOLOGÍA}

El objetivo fundamental de este trabajo es analizar los motivos que empujan (push factors) y atraen (pull factors) al turista a visitar la Feria Gastronómica y Artesanal Parque Natural Sierras de Cazorla, Segura y las Villas. La motivación se ha convertido en un aspecto de gran importancia pues se le considera detonante de la conducta humana y factor explicativo de la casi totalidad de los aspectos relacionados con la actividad turística. El conocer la motivación del visitante puede llegar a influir en la lealtad sobre el destino, elemento fundamental en las estrategias de marketing, al ser considerado el mejor estimador del comportamiento del consumidor (Chen y Chen, 2009). La lealtad del visitante se traduce una fuente de ingresos estables y mejora los beneficios del destino; y visitantes leales se convierten en un canal de información y comunicación positiva para otras personas (Baker y Crompton, 2000).

La investigación se basa en una encuesta realizada a una muestra representativa de los turistas asistentes a la segunda edición de la feria que cumplían dos condiciones de manera simultánea: primera, que se encontrasen visitando cualquiera de los establecimientos participantes en dicho festival culinario y, segundo, que ya hubiesen visitado, al menos, dos de dichos establecimientos. La encuesta se basa en trabajos previos relacionados con el turismo gastronómico (Ignatov y Smith, 2006; Okumus et al., 2007; McKercher et al., 2007; López-Guzmán y Sánchez Cañizares, 2012). Para la realización de la encuesta se utilizaron como encuestadores un grupo de especialistas en el ámbito turístico y en la atención al ciudadano con, al menos, el dominio de dos idiomas, para que hicieran la labor de encuestadores. En los días anteriores a la celebración del Festival Gastronómico, y debido a que ya existía un cierto número de turistas en la zona, se realizó un pre-test de 15 encuestas para detectar posibles desviaciones y errores, aplicando a la versión definitiva las mejoras detectadas en el mismo. En la cumplimentación de las encuestas, que eran totalmente anónimas, estaban presentes los encuestadores para resolver las dudas que pudieran surgir. La misma se distribuyó en dos idiomas (español e inglés). Se utilizó un muestreo de conveniencia, comúnmente utilizado en este tipo de investigaciones donde los encuestados están disponibles para ser encuestados en un espacio y tiempo determinado (Finn et al., 2000)

Los ítems utilizados en la encuesta pretenden dar respuesta a las medidas e indicadores propuestos para la realización del análisis de la demanda turística. El número de ítems fue de 20. Se utilizaron cuatro tipos de cuestiones: primero, preguntas basadas en una escala de Likert de 5 puntos para valorar la opinión del visitante; segundo, respuestas sí/no para obtener la percepción sobre determinados aspectos del viaje; tercero, cuestiones cerradas donde los turistas encuestados pudieron calificar esta experiencia gastronómica; cuarta, cuestiones para determinar el perfil sociodemográfico del visitante. La primera parte del cuestionario recoge preguntas relacionadas con el viaje (número de visitas previas al Parque Natural, tiempo de permanencia de la visita, tipo de establecimiento que utiliza para pernoctar, medio por el que ha tenido conocimiento del festival, etc.). Una 
segunda parte se centra en las motivaciones que atraen al turista al festival, los días que tiene previsto asistir y el presupuesto de gasto realizado o previsto. Un tercer bloque de preguntas reúne las impresiones sobre una batería de atributos relacionados con el evento, la mayor o menor propensión a consumir en su lugar de residencia productos gastronómicos de la feria, y sus intenciones de comportamiento futuro en función de la experiencia vivida. Y, finalmente, para completar el análisis se formularon un conjunto de preguntas de clasificación - género, edad, nivel de formación, etc. - al objeto de caracterizar a los visitantes. Un total de 269 cuestionarios válidos (de un total de 283 encuestas realizadas) fueron cumplimentados entre los días 3 y 4 de octubre de 2014 en el lugar de celebración del festival - Paraje de las Pistas Rojas en Pozo Alcón (Jaén) - en diferentes horarios del día. La tabulación y el tratamiento de los datos se han realizado a través del programa estadístico IBM SPSS Statistics 19. Debido a los pocos estudios existentes en torno a los festivales gastronómicos en la región de Andalucía no se dispone de un dato fiable sobre el número de turistas en este segmento con lo cual no podemos determinar una población objetiva.

Básicamente, los datos, resultados y conclusiones que se presentan en este artículo se refieren al análisis de la parte del cuestionario que hace referencia a las motivaciones o razones para asistir a la feria culinaria. La principal aportación tratar de dar respuesta a la pregunta: ¿cuál es la motivación del visitante que elige como destino turístico esta feria culinaria? La hipótesis a contrastar es si los turistas comparten o difieren significativamente en los motivos para asistir a la feria. Para ello se han utilizado diversas técnicas estadísticas: el análisis factorial, el análisis de conglomerados y el análisis de la varianza (ANOVA con comparaciones múltiples post-hoc). Cabe indicar que la investigación ha tenido como ámbito de aplicación una tipología concreta de destino turístico por lo que no resulta adecuado extrapolar los resultados a otros enclaves con características diferentes.

\section{RESULTADOS DE LA INVESTIGACIÓN Y DISCUSIÓN}

Este estudio parte inicialmente de un análisis descriptivo de las características sociodemográficas de la encuesta y análisis del comportamiento de los visitantes objeto de la muestra en relación visitas previas a Parque Natural Sierras de Cazorla, Segura y las Villas (Jaén), los días de permanencia en la comarca coincidiendo con la celebración de la feria, el tipo de establecimiento hotelero, la forma de viajar y medio por el que han conocido el festival, así como su repercusión económica. En una segunda etapa se analiza los motivos que determinaron la decisión de asistir para más tarde intentar identificar los distintos tipos de turistas que han visitado la feria, con el objeto de que estos resultados puedan servir para que los organizadores identifiquen al turista potencial para futuras ediciones, y a las administraciones para que tomen conciencia de la repercusión económica, tanto directa como indirecta, de este tipo de eventos.

Las características sociodemográficas de los encuestados (Tabla 1) revela una mayor proporción de mujeres $(54,2 \%)$ que de hombres (45,8\%), preferentemente en el tramo de edad comprendido entre los 40 y 49 años $(36,7 \%)$ y en menor proporción entre 30 y 39 años $(24,3 \%)$. Los asistentes muestran una alta formación académica, más de la mitad de los encuestados (54,5\%) es graduado universitario, frente a un $9,6 \%$ que tiene educación obligatoria o menos. Desde el punto de vista profesional, la mayoría son asalariados a tiempo completo $(23,7 \%)$ o funcionarios $(19,5 \%)$. Cabe destacar el bajo número de encuestados que reconocieron estar desempleados, sólo el $4,7 \%$, y de jubilados o pensionistas $(5,3 \%)$, frente a las estadísticas oficiales que sitúan cifras en niveles muy superiores. El turista visitante a la feria es básicamente andaluz $(83,4 \%)$ y en menor medida de la Comunidad de Madrid (10,7\%), siendo despreciable otros mercados emisores. A la pregunta si ha visitado con anterioridad el Parque Natural, el 49,1\% afirma que lo ha visitado anteriormente en más de tres ocasiones y un $29,0 \%$, entre una y tres veces. También existe un importante porcentaje de turistas $(21,9 \%)$ que afirman no haber venido con anterioridad, lo que convierte a la feria culinaria como reclamo turístico para Parque Natural. Su ubicación en un medio natural es un aliciente importante a la hora de decidir el viaje e incluso de prolongar la estancia de los visitantes. El 83,4\% de los turistas pernoctaron una o más noches, y de ellos, un $74,5 \%$ tenía previsto visitar la feria los dos días. Del total de pernoctaciones, sólo el $13,2 \%$ se aloja en hoteles, un $10,4 \%$ en camping y el $34,7 \%$ en alojamientos rurales; el restante $41,7 \%$ que lo hace en viviendas de amigos o familiares. Este evento culinario no atrae al turista familiar, tan sólo el $21,9 \%$ de los encuestados viene con hijos. La mayoría asiste sólo con la en pareja $(25,4 \%)$ o con amigos y compañeros de trabajo $(25,4 \%)$. En referencia al medio por el que han tenido referencia de la celebración del evento, el 60,9\% afirma que ha sido por recomendación de amigos o familiares; a gran distancia están los medios comunicación: folletos turísticos, redes sociales, agencias online; y la experiencia propia que apenas alcanzan el 10\%. Hay que resaltar el papel residual que desempeñan las agencias de viajes con oficina física en la proyección de este festival (2,4\%), lo que corrobora la tendencia dentro del sector turístico. Al objeto de evaluar el impacto económico de la feria, se preguntó la cantidad de dinero prevista gastar en la visita. El presupuesto de los encuestados oscila entre los 41 y $100 €$, situándose la mediana y la moda en el intervalo comprendido entre 41 y $60 €$ de presupuesto $(28,0 \%)$, mientras que el $20,2 \%$ está dispuesto a gastarse entre 81 y $100 €$ y el $18,5 \%$ entre 61 y $80 €$.

Uno de los principales objetivos del estudio es conocer los motivos que atraen a los turistas a la Feria Gastronómica y Artesanal del Parque Natural. La hipótesis a contrastar es si los turistas comparten o difieren significativamente en los motivos para asistir a una feria culinaria. Las razones o los motivos de la visita quedan reflejados en la octava pregunta del cuestionario y que se reproduce en tabla 2 de este artículo. Para ello se 
diseñó una escala en la que se trató de recoger las razones de viaje más frecuentes y relevantes analizados en investigaciones previas adaptándolas y teniendo en cuenta las características específicas del destino turístico y de los visitantes (McKercher et al., 2007; Yuan y Jang, 2008; Smith y Costello, 2009; Devesa et al., 2010; LópezGuzmán y Sánchez Cañizares, 2012; Gagic et al., 2013). Tras la realización de un pretest se seleccionaron 9 de los 11 ítems inicialmente planteados al objeto de determinar la importancia relativa de una serie de factores en su decisión de visitar el festival gastronómico. Se incluyeron factores internos y externos, tal y como establece la teoría de Crompton (1979) entre motivos de arrastre y motivos de empuje. El coeficiente alpha de Cronbach de la escala final alcanza un valor de 0,781 , lo que indica una consistencia interna meritoria entre los elementos de la escala. El índice de fiabilidad obtenido refuerza la validez del trabajo de investigación realizado (Nunnally y Bernstein, 1994).

Tabla 1: Características sociodemográficas de la muestra

\begin{tabular}{|c|c|c|}
\hline Variable & Categoría & $\begin{array}{l}\text { Porcentaje de } \\
\text { respuestas }\end{array}$ \\
\hline \multirow{2}{*}{ Género } & Masculino & $45,8 \%$ \\
\hline & Femenino & $54,2 \%$ \\
\hline \multirow{5}{*}{ Edad } & $<30$ años & $13,6 \%$ \\
\hline & 30-39 años & $24,3 \%$ \\
\hline & 40-49 años & $36,7 \%$ \\
\hline & 50-59 años & $16,6 \%$ \\
\hline & $\geq 60$ años & $8,9 \%$ \\
\hline \multirow{4}{*}{ Formación } & Enseñanza Obligatoria & $9,6 \%$ \\
\hline & Bachillerato/ F. P. & $35,9 \%$ \\
\hline & Graduado universitario & $41,9 \%$ \\
\hline & Postgrado & $12,6 \%$ \\
\hline \multirow{9}{*}{ Categoría profesional } & Profesional liberal & $8,9 \%$ \\
\hline & Empresario & $14,2 \%$ \\
\hline & Funcionario & $19,5 \%$ \\
\hline & Asalariado a tiempo completo & $23,7 \%$ \\
\hline & Asalariado a tiempo parcial & $8,3 \%$ \\
\hline & Autónomo & $8,9 \%$ \\
\hline & Estudiante & $5,3 \%$ \\
\hline & Desempleado & $4,7 \%$ \\
\hline & Jubilado/pensionista & $5,3 \%$ \\
\hline \multirow{6}{*}{ Renta disponible } & $<700 €$ & $8,4 \%$ \\
\hline & $700-1.000 €$ & $18,6 \%$ \\
\hline & $1.000-1.500 €$ & $40,7 \%$ \\
\hline & $1.500-2.500 €$ & $27,5 \%$ \\
\hline & $2.500-3.500 €$ & $1,8 \%$ \\
\hline & $+3.500 €$ & $3,0 \%$ \\
\hline
\end{tabular}

Fuente: Elaboración propia

Se ha realizado un análisis factorial de la escala de motivación para identificar un pequeño número de factores explicativos, extrayéndose tres dimensiones diferentes de la motivación en la Feria del Parque Natural Sierras de Cazorla, Segura y la Villas (Tabla 2). Si bien el interés reside en las puntuaciones factoriales que se derivan de dichos componentes como herramienta para establecer la fuerza de las motivaciones de cada visitante, resulta de utilidad caracterizar cada uno de los tres factores extraídos. El primer factor, denominado motivos hedónicos, relaciona a un turista que busca espacio y tiempo de disfrute lejos de las tensiones de la vida cotidiana, a la vez que vivir nuevas experiencias. El coeficiente alpha de Cronbach $(0,826)$ para los diferentes ítems que integran esta dimensión de motivación revela una alta consistencia de esta subescala. La importancia de este 
componente explica por sí solo un $28,6 \%$ de la varianza total de la matriz de motivaciones. El segundo factor encontrado, relacionado con motivos que se pueden denominar de conveniencia, corresponde a visitantes que buscan un destino próximo a su localidad de residencia y acorde a la economía familiar. El coeficiente alpha de Cronbach $(0,736)$ para esta subescala también muestra una buena consistencia interna. Este componente contribuye a explicar el $21,0 \%$ de la varianza de la matriz de motivaciones. Por último, el tercer factor extraído, denominado motivos gastronómicos, está relacionado con un turista que concibe la visita como un instrumento para conocer, degustar y poder adquirir los productos gastronómicos elaborados en el medio natural. El valor del coeficiente alpha de Cronbach $(0,689)$ constituye también una subescala fiable. Este componente explica un $20,4 \%$ de la varianza total de la matriz de motivaciones. Los resultados están en la línea con el núcleo de la teoría motivacional de Crompton (1979) que categoriza en dos grandes bloques los motivos que inciden sobre la conducta turística: motivos sociopsicológicos, donde el viaje o la visita es un medio para la satisfacción de necesidades de tipo social o psicológico de individuos o grupos, y motivos culturales, en los que la satisfacción obtenida estaría en relación con los atributos propios del destino turístico.

Tabla 2: Matriz factorial de componentes rotados - Motivación de la Visita -

\begin{tabular}{|c|c|c|c|c|}
\hline \multirow{2}{*}{ Variables motivaciones } & \multicolumn{3}{|c|}{ Componentes } & \multirow{2}{*}{$\begin{array}{l}\text { Dimensiones } \\
\text { Motivacionales }\end{array}$} \\
\hline & 1 & 2 & 3 & \\
\hline Conocer sitios nuevos & 0,840 & & & \multirow{3}{*}{ Hedónica } \\
\hline Desconectar de lo cotidiano & 0,809 & & & \\
\hline Pasar un día fuera & 0,787 & & & \\
\hline Cercanía al lugar de residencia & & 0,808 & & \multirow{3}{*}{ Conveniencia } \\
\hline Destino accesible al bolsillo & & 0,757 & & \\
\hline Ser una visita más del itinerario turístico & & 0,599 & & \\
\hline Degustar productos gastronómicos & & & 0,841 & \multirow{3}{*}{ Gastronómica } \\
\hline Conocer la gastronomía y artesanía de la zona & & & 0,774 & \\
\hline Realizar compras de interés & & & 0,699 & \\
\hline Autovalores & 3,350 & 1,850 & 1,093 & \\
\hline$\%$ varianza explicada & 28,558 & 21,005 & 20,358 & \\
\hline$\%$ varianza acumulada & 28,558 & 49,562 & 69,921 & \\
\hline KMO & \multicolumn{3}{|c|}{0,672} & \\
\hline Prueba de esfericidad de Barlett & \multicolumn{3}{|c|}{ Chi-cuadrado $=575,987$, sig $<0,01$} & \\
\hline
\end{tabular}

Fuente: Elaboración propia

El estudio de las motivaciones proporciona fundamentos para establecer una segmentación de la Feria del Parque Natural Sierras de Cazorla, Segura y la Villas como destino turístico. Para ello se ha efectuado un análisis de conglomerados no jerárquico con las puntuaciones factoriales de los dos factores. Bajo el criterio de maximizar la varianza entre tipologías y minimizar la varianza dentro de cada tipología, la mejor solución que cumple los criterios es la que establece cuatros conglomerados. La caracterización de los clústers a partir de las medias de las variables de motivación aparece en la Tabla 3. El estadístico F del ANOVA permite contrastar que las medias comparadas no son iguales, pero no permite precisar dónde se encuentran las diferencias detectadas. Para saber qué media difiere de otra, se ha utilizado un tipo particular de contraste denominado comparaciones múltiples post hoc o comparaciones a posteriori. Al objeto de efectuar estas comparaciones no puede asumirse que las varianzas poblaciones son iguales - el nivel crítico asociado al estadístico de Levene es menor que 0,05 para todos los casos, por lo que se rechaza la igualdad de varianzas -. El estadístico $\mathrm{F}$ del ANOVA se basa en el cumplimiento de dos supuestos: normalidad y homocedasticidad. Dado que no posible asumir que las varianzas poblacionales sean iguales, se recurre a los estadísticos de Brown-Forsythe y de Welch como alternativa al estadístico $\mathrm{F}$ del ANOVA -Tabla 4 -. Puesto que el nivel crítico asociado ambos estadísticos es menor que 0,05 , se puede rechazar la hipótesis de igualdad de medias y concluir que los promedios de las variables motivacionales de los tres conglomerados comparados no son iguales. Para poder contrastar las diferencias significativas entre las diferentes medias se ha aplicado la prueba Games-Howell.

El primero de los clúster presenta está integrado por un 18,8\% de los encuestados y muestra valores significativos en casi todas las variables - en ocho de las nueve consideradas -. Es el segmento que más bajos 
registros muestra en las variables relacionadas con la motivación culinaria o gastronómica. Otro dato importante es el hecho que registre bajas puntuaciones en el resto de las variables, lo que implica que no relacione claramente con alguno de los ámbitos motivacionales extraídos. Por todo ello, este segmento podría corresponder a un visitante con otras motivaciones turísticas o no turísticas y que el estudio realizado no ha podido detectar. El segundo de los clúster está integrado por el $23,8 \%$ de los encuestados, los valores más altos con diferencias significativas se observan en las motivaciones conocer sitios nuevos y desconectar de lo cotidiano. Se caracteriza por ser un turista que además de disfrutar de la gastronomía se plantea la visita para romper con la rutina y escapar de las tensiones. Al relacionar claramente con las dimensiones primera y tercera el análisis factorial a este segmento se le ha denominado visitante hedónico-gastronómico. El tercer de los conglomerados se caracteriza por mostrar los registros más elevados en ocho de las nueve variables consideradas, es el segmento más numeroso al integrar el $41,9 \%$ de los turistas. Los valores más elevados con diferencias significativas se observan en todas las variables no relacionadas con la motivación culinaria. Con diferencia, es el segmento que más valora que la visita esté próxima al lugar de residencia y sea barata para el bolsillo. La clara relación con todas las dimensiones motivacionales permite considerar que estamos ante un visitante multimotivado. Por último, el cuarto segmento lo integra el 15,5\% de los visitantes, siendo el menos numeroso y el que más bajo puntúa en todas las variables no relacionas con la gastronomía. Se caracteriza por ser un turista que se plantea exclusivamente la visita para disfrutar de los atributos propios del destino turístico, o sea, la gastronomía.

Tabla 3: Caracterización clúster a partir de las medias de las variables de motivación

\begin{tabular}{|c|c|c|c|c|c|c|c|c|}
\hline \multirow{3}{*}{ Variables motivacionales } & \multicolumn{4}{|c|}{ Clúster de pertenencia } & \multirow{2}{*}{\multicolumn{2}{|c|}{ ANOVA }} & \multirow{2}{*}{\multicolumn{2}{|c|}{$\begin{array}{l}\text { Estadístico } \\
\text { igualdad de } \\
\text { varianzas }\end{array}$}} \\
\hline & \multirow{2}{*}{$\frac{1}{\text { Media }}$} & \multirow{2}{*}{$\frac{2}{\text { Media }}$} & \multirow{2}{*}{$\frac{3}{\text { Media }}$} & \multirow{2}{*}{$\begin{array}{c}4 \\
\text { Media }\end{array}$} & & & & \\
\hline & & & & & $\mathbf{F}$ & Sig. & Levene & Sig. \\
\hline Conocer sitios nuevos & $2,53^{(*)}$ & $4,37^{(*)}$ & $4,34^{(*)}$ & $1,08^{(*)}$ & 71,152 & $<0,01$ & 23,140 & $<0,01$ \\
\hline Desconectar de lo cotidiano & $2,67^{(*)}$ & $4, \mathbf{0 3}^{(*)}$ & $4,15^{(*)}$ & $1,72^{(*)}$ & 23,460 & $<0,01$ & 1,883 & $<0,13$ \\
\hline Pasar un día fuera & $2,90^{(*)}$ & 3,74 & $\left.4,09^{(*)}\right)$ & $1,00^{(*)}$ & 37,865 & $<0,01$ & 18,215 & $<0,01$ \\
\hline Cercanía lugar de residencia & $3,07^{(*)}$ & $1,47^{(*)}$ & $4,16^{(*)}$ & 2,16 & 39,436 & $<0,01$ & 7,568 & $<0,01$ \\
\hline Destino accesible al bolsillo & 2,93 & 2,74 & $4,72^{(*)}$ & 2,28 & 44,586 & $<0,01$ & 24,421 & $<0,01$ \\
\hline Ser una visita más del itinerario & $2,47^{(*)}$ & $2,74^{(*)}$ & $4,48^{(*)}$ & $1,40^{(*)}$ & 52,266 & $<0,01$ & 8,511 & $<0,01$ \\
\hline Degustar productos gastronómicos & $3,40^{(*)}$ & 4,82 & 4,81 & 4,96 & 45,358 & $<0,01$ & 24,872 & $<0,01$ \\
\hline Conocer la gastronomía y artesanía & $2,67^{(*)}$ & 4,68 & 4,79 & 4,88 & 80,405 & $<0,01$ & 13,450 & $<0,01$ \\
\hline Realizar compras de interés & $2,90^{(*)}$ & $3, \mathbf{4 5} 5^{(*)}$ & $4,55^{(*)}$ & $4,68^{(*)}$ & 32,947 & $<0,01$ & 6,147 & $<0,01$ \\
\hline
\end{tabular}

$\left(^{*}\right)$ Los valores en negrita presentan diferencias significativas con al menos dos de las medias de los cuatro conglomerados en análisis post-hoc del ANOVA

Fuente: Elaboración propia.

Tabla 4: Pruebas robustas igualdad de medias

\begin{tabular}{|c|c|c|c|c|c|}
\hline \multicolumn{2}{|c|}{ Variables motivacionales } & Estadístico ${ }^{\mathrm{a}}$ & gl1 & gl2 & Sig. \\
\hline \multirow{2}{*}{ Conocer sitios nuevos } & Welch & 225,261 & 3 & 75,179 & $<0,01$ \\
\hline & Brown-Forsythe & 75,013 & 3 & 76,026 & $<0,01$ \\
\hline \multirow{2}{*}{ Desconectar de lo cotidiano } & Welch & 22,932 & 3 & 68,110 & $<0,01$ \\
\hline & Brown-Forsythe & 23,509 & 3 & 113,790 & $<0,01$ \\
\hline \multirow{2}{*}{ Pasar un día fuera } & Welch & . & . & . & . \\
\hline & Brown-Forsythe & . & . & . & . \\
\hline \multirow{2}{*}{ Cercanía lugar de residencia } & Welch & 54,854 & 3 & 64,749 & $<0,01$ \\
\hline & Brown-Forsythe & 34,898 & 3 & 88,629 & $<0,01$ \\
\hline Destino accesible al bolsillo & Welch & 54,595 & 3 & 52,427 & $<0,01$ \\
\hline
\end{tabular}




\begin{tabular}{|c|c|c|c|c|c|}
\hline & Brown-Forsythe & 32,992 & 3 & 86,629 & $<0,01$ \\
\hline \multirow{2}{*}{ Ser una visita más del itinerario } & Welch & 62,988 & 3 & 61,199 & $<0,01$ \\
\hline & Brown-Forsythe & 44,937 & 3 & 102,025 & $<0,01$ \\
\hline \multirow{2}{*}{ Degustar productos gastronómicos } & Welch & 18,851 & 3 & 71,000 & $<0,01$ \\
\hline & Brown-Forsythe & 37,194 & 3 & 48,364 & $<0,01$ \\
\hline \multirow{2}{*}{ Conocer la gastronomía y artesanía } & Welch & 33,362 & 3 & 71,212 & $<0,01$ \\
\hline & Brown-Forsythe & 71,297 & 3 & 55,641 & $<0,01$ \\
\hline \multirow{2}{*}{ Realizar compras de interés } & Welch & 25,481 & 3 & 60,920 & $<0,01$ \\
\hline & Brown-Forsythe & 28,085 & 3 & 89,900 & $<0,01$ \\
\hline \multicolumn{6}{|c|}{$\begin{array}{l}\text { a. Distribuidos en } \mathrm{F} \text { asintóticamente. } \\
\text { b. No se pueden realizar las pruebas robustas de la igualdad de medias para pasar un día fuera porque al } \\
\text { menos un grupo tiene varianza } 0 \text {. }\end{array}$} \\
\hline
\end{tabular}

Fuente: Elaboración propia.

\section{CONCLUSIONES, LIMITACIONES Y FUTURAS LÍNEAS DE INVESTIGACIÓN}

Una feria sobre la gastronomía y artesanía de una localidad rural tiene como objetivos principales dar a conocer sus productos y la creación de marca. Su éxito se mide principalmente por el número de turistas que acuden al evento, por el dinero que se gastan, pero en las primeras ediciones de este tipo de festivales no consolidados un factor determinante es la experiencia o la satisfacción real que estos turistas puedan tener (Stilling y Halkier, 2014). En este trabajo se analizan las principales motivaciones del turista para asistir a la "Il Feria Gastronómica y Artesanal del Parque Natural Sierras de Cazorla, Segura y la Villas" celebrada los días 3 y 4 de octubre de 2014 en la localidad de Pozo Alcón (España), considerando este evento dentro de lo que se conoce globalmente como gastroturismo, que abarca los viajes realizados por personas para comprar o consumir productos regionales. El análisis efectuado ha permitido identificar tres factores motivacionales diferentes: motivaciones gastronómicas, motivaciones hedonísticas y, por último, motivos de conveniencia. En base a ellos se han establecido cuatro tipologías de visitantes - turista multimotivado, turista hedónico-gastronómico, turista gastronómico y el turista con otras motivaciones - que se consideran como válidas y de utilidad para segmentar el mercado.

Los resultados obtenidos tienen importantes implicaciones para la planificación y gestión de futuras ediciones de la Feria Gastronómica y Artesanal del Parque Natural Sierras de Cazorla, Segura y la Villas. Al ser la gastronomía la variable principal que motiva la asistencia a la feria, futuras ediciones tendrán éxito en la medida en que se aumente el valor de "marca" para los productos locales del Parque Natural. Hay que tener muy presente que el resultado final de las visitas tienen asociaciones de satisfacción con la comarca y pueden estimular su divulgación boca-a-boca e incrementar el número de turistas que vuelvan a ediciones futuras. Determinar qué características tienen estos visitantes, qué factores les han motivado para asistir al evento y satisfacer esas necesidades en futuras ediciones, determinarán su éxito a largo plazo. De los resultados de la investigación se deduce que la mayoría de los encuestados no han tenido otra información que la comunicación a través de sus allegados, por lo que sería necesario incrementar la publicidad de la feria y establecer mejores canales de comunicación, básicamente en las provincias cercanas - Jaén, Córdoba y Granada - que son el mercado origen de los visitantes, además de ser una de las dimensiones motivacionales detectadas conveniencia -. Los promotores deben tener presente que la mayor parte de los visitantes son de edad madura, que preferentemente asisten en compañía de amigos o pareja y con un presupuesto de gasto medio-alto, siendo el segmento más numeroso el que tiene un carácter multimotivacional. Este grupo junto al segmento hedónicogastronómico - representan el $65,7 \%$ de los visitantes -, además de la gastronomía, comparten un componente lúdico y de entretenimiento importante, por lo que lo factor gastronómico debe saber coexistir y adaptarse. En este sentido es necesario garantizar el acomodo del visitante para facilitar no sólo la compra, sino también la degustación de los productos dentro del propio recinto. Luego para el enfoque de promoción de los productos gastronómicos y artesanos, se deben presentar expositores de calidad, con talleres educativos lúdicos sobre la gastronomía y la artesanía que pongan en valor los productos de la comarca, la posibilidad de consumir, en el mismo recinto, estos productos elaborados por profesionales, mientras se disfruta de otras actividades que puedan satisfacer la necesidad de divertimento del turista y del goce de los sentidos.

Las limitaciones al presente estudio están relacionadas con la localización de la feria, una localidad rural enclavada en un parque natural, realizándose las entrevistas dentro del recinto, lo que hace que pueda causar sesgo sobre la motivación de asistencia a este tipo de eventos y presenten problemas en la generalización del comportamiento de los asistentes. En cuanto a las futuras líneas de investigación se propone profundizar en el 
análisis de las diferencias motivaciones de los turistas en función de sus características sociodemográficas, forma de organizar el viaje, etc. De igual forma, también se platea como futura línea de investigación un estudio del impacto económico de la feria, así como la mayor o menor capacidad que pueda tener como instrumento de promoción turística del Parque Natural Sierras de Cazorla, Segura y la Villas. A pesar de estas limitaciones, los resultados presentados en este estudio contribuyen a completar la literatura académica en este campo, proporcionando una mejor comprensión de los festivales y ferias de turismo gastronómico y artesanal.

\section{BIBLIOGRAFÍA}

Baker, D. A. y Crompton, J. L. (2000). Quality, satisfaction and behavioural intentions. Annals of Tourism Research, 27 (3), 785-804.

Boniface, P. (2003). Tasting tourism: travelling for food and drink. Aldershot: Ashgate Publishing Limited.

Chen, C. F. y Chen, F. S. (2009). Experience quality, perceive value, satisfaction, and behavioral intentions for heritage tourists. Tourism Management, 31 (1), 29-35.

Cohen, E. y Avieli, N. (2004). Food in tourism: attraction and impediment. Annals of Tourism Research, 31 (4), 755-778.

Cracolici, M. F. y Nijkamp, P. (2008). The attractiveness and competitiveness of tourist destinations: A study of Southern Italian regions. Tourism Management, 30, 336-344.

Crompton, J. L. (1979). Motivations for pleasure vacation. Annals of Tourism Research, 6 (4), 408-424.

Devesa, M., Laguna, M. y Palacios, A. (2010). The role of motivation in visitor satisfaction: Empirical evidence in rural tourism. Tourism Management, 31, 547-552.

Du Rand, G. E., Heath, E. y Alberts, N. (2003). The role of local and regional food in destination marketing: A South African situation analysis. Journal of Travel and Tourism Marketing, 14 (3/4), 97-112.

Finn, M., Elliott-White, M. y Walton, M. (2000). Tourism and leisure research methods: Data collection, analysis and interpretation. Harlow: Pearson Education.

Gagic, S. M., Tesanovi, D. V., Ivokoz-Dzigurski, A. C., Pivac, T. D. y Jovici, A. D. (2013). Motives and attitudes of food and drink festival visitors: A case study of Serbia. Journal of Food, Agriculture \& Environment, 11 (1), 1055-1059.

Hall, C. M., Sharples, L., Cambourne, B. y Macionis, N. (2000): Wine tourism around the world: development, management and market. Oxford: Elsevier.

Hall, C. M., Sharples, L., Mitchell, R., Macionis, N. y Cambourne, B. (2003). Food tourism around the World. Oxford: Butterworth-Heinemann.

Hjalager, A. M. y Richards, G. (2002). Tourism and Gastronomy. Londres: Routledge.

Ignatov, E. y Smith, S. (2006). Segmenting Canadian culinary tourist. Current Issues in Tourism, 9 (3), $235-255$.

Kneafsey, M. (2007). Tourism, place identities and social relations in the European rural periphery. European Urban and Regional Studies, 7 (1), 35-50.

Long, L. (2003). Culinary Tourism. Lexington: University of Kentucky Press.

López-Guzmán, T. y Sánchez-Cañizares, S. (2012). Culinary tourism in Cordoba (Spain). British Food Journal, 114 (2), 168-179.

Marko, S., Veljko, C., Veljko, D., Čedomir, A., Dragan, T. y Đorde, A. (2013). Motives and attitudes of food and drink festival visitors: A case study of Serbia. Journal of Food, Agriculture \& Environment, 11 (1), 10551059.

McKercher, B., Okumus, F. y Okumus, B. (2008). Food tourism as a viable market segment: It's all how you cook the numbers!. Journal of Travel and Tourism Marketing, 25 (2), 137-148.

Nunnally, J. C. y Bernstein, I. H. (1994). Psychometric theory. Nueva York: McGraw-Hill.

Okumus, B., Okumus, F. y McKercher, B. (2007). Incorporating local and international cuisines in the marketing of tourism destinations: the cases of Hong Kong and Turkey. Tourism Management, 28, 253-261.

Oliveira, S. (2007). La importancia de la gastronomía en el turismo. Un ejemplo de Mealhada. Estudios y Perspectivas en Turismo, 16 (3), 26-282.

Quan, S. y Wang, N. (2004). Towards a structural model of the tourist experience: an illustration from food experiences in tourism. Tourism Management, 25, 297-305.

Riley, M. (2005). Food on beverage management. A review of change, International Journal of Contemporary Hospitality Management, 17 (1), 88-93. 
Schlüter, R. G. (2003). Turismo y patrimonio gastronómico. Una perspectiva. Buenos Aires: Centro de Investigaciones y Estudios Turísticos.

Sedmark, G. y Mihalic, T. (2008). Authenticity in mature seaside resorts. Annals of Tourism Research, 35, 10071031.

Sims, R. (2009). Food, place and authenticity: Local food and the sustainable tourism experience. Journal of Sustainable Tourism, 17 (3), 321-336.

Smith, S. y Costello, C. (2009). Segmenting visitors to a culinary event: motivations, travel behaviour, and expenditures, Journal of Hospitality Marketing \& Management, 18 (1), 44-67,

Stilling, B. y Halkier, H. (2014). Mussels, Tourism and Community Development: A Case Study of Place Branding Through Food Festivals in Rural North Jutland, Denmark. European Planning Studies, 22 (8), 1587-1603

Tikkanem, I. (2007). Maslow's hierarchy and food tourist in Finland: five cases. British Food Journal, 109 (9), 721 734.

Tse, P. y Crotts, J. C. (2005). Antecedents of novelty seeking: International visitors propensity to experiment across Hong Kong's culinary traditions. Tourism Management, 26, 965-968.

Yuan, J. y Jang, S. (2008). The effects of quality and satisfaction on awareness and behavioral intentions: exploring the role of a wine festival. Journal of Travel Research, 46, 279-288. 